\title{
Dinámica de Phragmites australis y Schoenoplectus AMERICANUS EN RESPUESTA A LA ADICIÓN DE FÓSFORO Y NITRÓGENO EN HUMEDALES EXPERIMENTALES
}

\author{
Yazmín Escutia-LaRa ${ }^{1,3}$ y Roberto Lindig-Cisneros ${ }^{2}$ \\ ${ }^{1}$ Facultad de Biología. Universidad Michoacana de San Nicolás de Hidalgo, Michoacán, México. \\ ${ }^{2}$ Centro de Investigaciones en Ecosistemas. Universidad Nacional Autónoma de México, Michoacán, México. \\ ${ }^{3}$ Autora para la correspondencia: yazminelb@yahoo.com.mx
}

\begin{abstract}
Resumen: Las especies invasoras, como Phragmites australis, resultan grandes competidoras para las especies nativas; sin embargo, es poco lo que se conoce sobre el mecanismo de invasión. En este trabajo se evaluó la interacción de $P$. australis con una especie nativa, Schoenoplectus americanus, mediante un experimento de parcelas divididas con adición de nutrimento (nitrógeno y fósforo). Para $P$. australis se encontraron diferencias $(P<0.05)$ entre tratamientos para la altura, el número de tallos y la biomasa aérea y de rizomas, mientras que para $S$. americanus se encontraron diferencias $(P<0.05)$ en la altura máxima, la altura promedio de tallos, el número de tallos, el porcentaje de cobertura y la biomasa aérea. El incremento de nutrimentos no permitió que $P$. australis desplazara a $S$. americanus, lo que sugiere que en condiciones naturales $S$. americanus será capaz de competir por los nutrimentos siempre y cuando no se presente algún disturbio, ya que en este estudio se mantuvo el dosel de especies nativas antes de introducir a $P$. australis. Los resultados sugieren que la competencia intraespecífica es más fuerte que la interespecífica en ausencia de disturbio.
\end{abstract}

Palabras clave: competencia, composición, disturbio, diversidad, nutrimentos, restauración ecológica.

\begin{abstract}
Invasive species, such as Phragmites australis, are major competitors for native species, however, very little is known about the mechanisms of invasion. In this study the interaction between $P$. australis with a native species, Schoenoplectus americanus, was evaluated through a split-plot experiment with addition of nutrients (nitrogen and phosphorus). For P. australis statistically significant differences $(P<0.05)$ were found among treatments for height, number of stems, aboveground biomass and rhizomes, while for $S$. americanus, statistically significant differences $(P<0.05)$ were found for maximum height, average height of stems and number of stems, percentage of coverage and aboveground biomass. The increase in nutrients did not allowed the displacement of S. americanus by P. australis. This result suggest that under natural conditions S. americanus could be able to compete for nutrients and not be displaced by $P$. australis, as longs as no disturbance occurs, because in this study the native species canopy was not altered before introducing $P$. australis. Our results suggest that intraspecific competition is stronger than interspecific in the absence of disturbance.
\end{abstract}

Key words: competence, composition, disturbance, diversity, ecological restoration, nutriments.

$\mathbf{L}$ os humedales son ecosistemas que tienen sustratos saturados e incluyen plantas que dependen de las condiciones propias de los suelos hídricos (Mitsch y Gosselink, 1993). El estudio del efecto del impacto humano en los humedales es de gran importancia debido a los servicios ecosistémicos que proporcionan y su valor para mantener la biodiversidad (León et al., 1995; Young, 1998; Montoya, 1984; Lloyd-Reilley y Maher, 2011). La calidad y cantidad del agua son factores que determinan el estado de estos eco- sistemas (Young, 1998); por lo que la hidrología y la disponibilidad de nutrimentos son dos de los principales factores que inciden en la composición de la vegetación en muchos humedales de agua dulce (Gosselink y Turner, 1978). Por ejemplo, la profundidad del agua y el hidroperíodo determinan la distribución de las especies vegetales en los humedales, ya que afectan directamente la dinámica poblacional al afectar la germinación, la supervivencia de las plantas y su éxito reproductivo (van der Valk y Davis, 1978). 
Por otro lado, los niveles de nutrimentos alteran las relaciones de competencia entre las especies vegetales, en particular si los taxones nativos están adaptados a la supervivencia en condiciones de bajos niveles de nutrimentos y los taxones invasores son capaces de mostrar crecimiento rápido cuando se elevan estos niveles (Verhoeven et al., 1993; Miao y Bazzaz, 1990; Verhoeven et al., 1996). La sensibilidad ambiental de algunas especies a diversas condiciones de nutrimentos y disponibilidad de agua, a menudo depende de la fase de desarrollo de las mismas. Por ejemplo, la germinación de muchas especies de humedales es inhibida por condiciones de inundación prolongada (Moore y Keddy, 1988).

Las invasiones por especies no nativas causan gran preocupación entre los responsables del manejo de áreas naturales por sus efectos ecosistémicos negativos (Mack et al., 2000); y, en particular, porque muchas invasiones son causadas por factores antropogénicos, tales como la perturbación y la eutrofización (D'Antonio, 1993; Alpert et al., 2000; Thompson et al., 2001). Sin embargo, indistintamente de la causa que lleva a que una planta se convierta en invasora, pueden llegar a alterar las interacciones entre especies y las condiciones ambientales (Vitousek et al., 1987; D’Antonio y Vitousek, 1992; Gordon, 1998), dando como resultado cambios en la composición de las comunidades y pérdidas en la biodiversidad.

Las interacciones de competencia entre plantas pueden ser determinadas por la composición de las comunidades vegetales, pues la presencia de especies nativas con capacidad competitiva o la propia composición de la comunidad pueden alterar el resultado de la interacción (Reader y Bonser, 1993; Howard y Goldberg, 2001; Lindig-Cisneros y Zedler, 2002a, b). Las especies invasoras son grandes competidoras para las nativas, llegando las primeras a formar rodales o manchones monoespecíficos al desplazar a las segundas (Bruce et al., 1995; Sanleón et al., 1999; Rogers y Hartemink, 2000).

En los estudios de competencia inter e intraespecífica, en donde se modifican las condiciones físicas bajo las que ocurre la interacción, no siempre se observa el mismo patrón, donde la especie invasora desplaza a la nativa. Por ejemplo, las especies invasoras tienen fuertes efectos intraespecíficos sobre la competencia interespecífica, variando las proporciones en experimentos con plantas invasoras no nativas y nativas que crecen juntas (Huenneke y Thomson, 1995; Weihe y Neely, 1997; Meekins y McCarthy, 1999). Existe evidencia de que la competencia intraespecífica es más fuerte que la interespecífica para especies invasoras, a pesar de lo cual son capaces de desplazar a especies nativas (Bakker y Wilson, 2001). Cuando se evalúa la interacción de plántulas en condiciones controladas (en macetas) se han detectado mayores efectos de competencia a nivel interespecífico (Gaudet y Keddy, 1995; Nernberg y Dale, 1997).

Diversos estudios sugieren que el efecto de la compe- tencia se relaciona con el tamaño individual de cada especie (Goldberg, 1987; Peltzer y Köchy, 2001; Weigelt et $a l ., 2002)$ y con la cercanía filogenética de las especies que coexisten (Goldberg, 1990). Varios autores han demostrado que la producción y almacenamiento de nutrimentos en las raíces y brotes de Phragmites australis aumentan con el incremento de sus concentraciones en el suelo (Ulrichy Burton, 1985; Boar, 1996). La distribución cosmopolita de P. australis refleja su capacidad para adaptarse a una amplia gama de ambientes (Ksenofontova, 1988). Esta capacidad de $P$. australis para tolerar diferentes niveles de nutrimentos es una de las razones por las que esta especie se ha estudiado con detalle en humedales artificiales (Berendse y Aerts, 1987; Aerts y De Caluwe, 1994; Aerts y Chapin, 1999).

Schoenoplectus americanus (Pers.) Volkart ex Schinz \& R.Séller, anteriormente llamada Scirpus americanus, conocida comúnmente como "junco", es una planta frecuente en los humedales, perteneciente a la familia Cyperaceae. Estudios en ciperáceas como S. mucronatus (L.) Pall. subsp. robustus (Miq.) T.Koyama, demuestran que el nitrógeno y el fósforo son los elementos fundamentales en la dinámica de crecimiento de angiospermas acuáticas como éstas (Hwang et al., 1996; Hwang et al., 2000). Los estudios en S. americanus han demostrado que la variación de absorción de los nutrimentos aumenta únicamente en el periodo de crecimiento (Boyd, 1970; Lloyd-Reilley y Maher, 2011).

En los humedales de Michoacán, Schoenoplectus americanus generalmente se encuentra junto con Typha domingesis y T. latifolia. Las especies de Typha se distribuyen al bordo del espejo de agua y a medida que el nivel del agua se encuentra más cercano a la superficie del suelo o incluso debajo de éste se presenta $S$. americanus. En un experimento de coexistencia entre $T$. domingensis y $S$. americanus (Escutia-Lara et al., 2009) se evaluó el efecto de diferentes concentraciones de nitrógeno y fósforo, así como la combinación de ambos nutrimentos, en el desempeño de las especies. En ese experimento, el tratamiento más favorable para $S$. americanus fue la adición de nitrógeno más fósforo, ya que causó un aumento en la densidad de los tallos. La adición de únicamente de fósforo tuvo el mismo efecto pero en menor magnitud, mientras que el nitrógeno por sí solo no tuvo un efecto positivo en el crecimiento de $S$. americanus (Escutia-Lara et al., 2009). En otro experimento se encontró que la adición de nitrato incrementa la acumulación de biomasa aérea y en rizomas, la altura y número de retoños de $T$. domingensis, $S$. americanus y Phragmites australis, mientras que el fosfato sólo tuvo efecto en el desarrollo de la biomasa aérea de $S$. americanus (Martínez de la Cruz, 2010).

La dinámica del proceso de invasión de Phragmites australis en humedales del occidente de México es poco conocida, por lo que se evaluó el desarrollo de $P$. australis en comunidades artificiales formadas por especies nativas características de esta zona del país. Se estableció la hipótesis 
de que $P$. australis desplaza más rápidamente a las especies nativas cuando se incrementan las concentraciones de nutrimentos en el medio.

\section{Materiales y métodos}

Se prepararon 36 unidades experimentales en abril del 2008, en las instalaciones del Centro de Investigaciones en Ecosistemas (CIEco), Universidad Nacional Autónoma de México (UNAM), Campus Morelia, bajo condiciones controladas. Cada unidad experimental consistió en un recipiente con un volumen de 210 -L $(100 \times 68 \times 40 \mathrm{~cm})$ y un área superficial de $0.58 \mathrm{~m}^{2}$. Se llenaron con una mezcla de tierra localmente colectada y turba comercial, en proporción 1:1 previamente homogeneizada. Cada contenedor contó con una válvula de flotación para mantener el nivel del agua constante y a la misma altura con respecto a la superficie del sustrato contenida en la unidad experimental.

En cada recipiente se plantaron núcleos de suelo con plantas nativas procedentes del humedal del manantial de la Mintzita. Los núcleos estaban dominados por Schoenoplectus americanus, sin embargo, también presentaban Eleocharis montevidensis, Hidrocotyle verticillata, Sagittaria latifolia y en menor medida Cyperus niger y Polygonum hydropiperoides. Se favoreció su establecimiento y desarrollo por un año, considerando que en experimentos previos este tiempo fue suficiente para lograr una cobertura del 100\% (Escutia-Lara, 2008). Al inicio de la segunda tem- porada de crecimiento en cada unidad experimental se plantó un rizoma de Phragmites australis con peso promedio de $29.47 \pm 7.7$ para evaluar su interacción con las especies nativas.

Para determinar las dosis de fertilización se utilizaron los resultados de un experimento previo (Escutia-Lara et al., 2010). Se inició la fertilización en el mes de abril de 2009. El diseño experimental consistió en un factorial de tres tratamientos con doce repeticiones cada uno incluyendo el testigo, donde las unidades experimentales quedaron distribuidas al azar. Las dosis de fertilizantes fueron: $0.00 \mathrm{~g}$ para el testigo (control), tratamiento $2\left(\mathrm{KNO}_{3}(18 \mathrm{~g})+\mathrm{KH}-\right.$ ${ }_{2} \mathrm{PO}_{4}(10.35 \mathrm{~g})$ por semana durante seis meses y tratamiento $3\left(\mathrm{KNO}_{3}(18 \mathrm{~g})+\mathrm{KH}_{2} \mathrm{PO}_{4}(10.35 \mathrm{~g})\right.$ por semana durante seis meses en el primer año y repitiendo esta dosis en el segundo año. El efecto del potasio no se consideró porque no se establecieron tratamientos en función de este nutrimento, las cantidades aportadas son suficientes para que no haya deficiencia por este elemento y es sabido que este elemento no causa efectos tóxicos en las plantas aun en altas concentraciones (Núñez-Escobar, 2007).

Una vez terminada la segunda fertilización se cosechó la biomasa aérea de Schoenoplectus americanus de la mitad de cada uno de los tratamientos, contando así con seis réplicas de cada tratamiento cortado y sin cortar, se continuo la evaluación por seis meses más para finalizar el experimento en abril del 2011, cosechándose la biomasa aérea de Phragmites australis y sus rizomas.

Cuadro 1. Especies registradas en las unidades experimentales de abril de 2009 a octubre de $2010 \quad X=$ Presencia, $\quad *=$ Solo en una unidad experimental, $\quad * *=$ En dos unidades experimentales, $>=$ En tres unidades experimentales.

\begin{tabular}{|c|c|c|c|c|c|c|c|c|c|c|c|c|c|c|c|c|c|c|c|}
\hline Año & & & & 200 & & & & & & & & 2010 & & & & & & & \\
\hline Especies/mes & A & M & $J$ & J & A & $S$ & $\mathrm{O}$ & $N$ & $\mathrm{D}$ & $\mathrm{E}$ & $\mathrm{F}$ & M & A & M & $J$ & $J$ & A & $S$ & $\mathrm{O}$ \\
\hline Berula erecta & & & * & $*$ & & & & & * & $*$ & & & $*$ & * & $*$ & * & * & $*$ & * \\
\hline Bidens aurea & $*$ & & & & & & & & & & & & & & & & & & \\
\hline Cyperus esculentus & & & $X$ & $x$ & $x$ & $x$ & & & & & & & & & & & & & \\
\hline Cyperus niger & $x$ & $X$ & $X$ & $x$ & $X$ & $x$ & $x$ & $x$ & & & $x$ & $x$ & $X$ & $x$ & $X$ & $x$ & $X$ & $X$ & $\mathrm{X}$ \\
\hline Eleocharis montevidensis & $x$ & $x$ & $x$ & $x$ & $x$ & $x$ & $x$ & $x$ & $* *$ & $* *$ & $\mathrm{x}$ & $x$ & $x$ & $x$ & $x$ & $x$ & $x$ & $x$ & $x$ \\
\hline Epilobium ciliatum & * & $x$ & $x$ & $x$ & $x$ & & & & * & * & & & $x$ & $x$ & $x$ & $x$ & & & \\
\hline Eupatorium rugosum & $x$ & $x$ & $x$ & $x$ & $x$ & $x$ & $* *$ & & & $x$ & $x$ & $x$ & $x$ & $x$ & $x$ & $x$ & $>$ & $>$ & $>$ \\
\hline Galium trifidum & & $x$ & * & * & * & & & & & & & & & * & $*$ & $*$ & & & \\
\hline Gnaphalium americanum & & & $x$ & $x$ & $x$ & & & & & & & & & $X$ & $X$ & $x$ & $X$ & & \\
\hline Hydrocotyle verticillata & $x$ & $x$ & $x$ & $x$ & $x$ & $x$ & $x$ & $x$ & $x$ & $x$ & $\mathrm{x}$ & $x$ & $X$ & $x$ & $x$ & $x$ & $x$ & $X$ & $\mathrm{X}$ \\
\hline Lythrum salicaria & & & $>$ & $>$ & & * & & & & & & & & $>$ & $>$ & $>$ & * & $*$ & \\
\hline Mimulus glabratus & & $x$ & * & $x$ & * & & & & & & & & & $* *$ & $* *$ & $* *$ & & & \\
\hline Poaceae 1 (Gramineae) & $x$ & $x$ & $X$ & $x$ & $X$ & $x$ & $x$ & & & & $x$ & $x$ & $X$ & $x$ & $X$ & $x$ & $X$ & $X$ & $\mathrm{X}$ \\
\hline Poaceae 1 (Gramineae) & $x$ & $x$ & $x$ & $x$ & $x$ & $x$ & $x$ & & & & $\mathrm{x}$ & $x$ & $x$ & $x$ & $x$ & $x$ & $x$ & $X$ & $x$ \\
\hline Polygonum hydropiperoides & & & * & & $* *$ & & & & & & & $<$ & $<$ & $<$ & $<$ & $<$ & & & \\
\hline Sagittaria latifolia & & $x$ & $x$ & $x$ & $x$ & $x$ & & & $x$ & $x$ & $x$ & $x$ & $x$ & $x$ & $x$ & $x$ & $x$ & & \\
\hline Solanum americanum & & & * & * & $x$ & & & & & & & & * & * & $*$ & & & & \\
\hline
\end{tabular}


Yazmín Escutia-Lara y Roberto Lindig-Cisneros

Cuadro 2. Especies registradas en las unidades experimentales por tratamiento de abril de 2009 a octubre de $2010 \quad X=$ Presencia

\begin{tabular}{|c|c|c|c|c|c|c|}
\hline Año & 2009 & & 2010 & & & \\
\hline Especies / Tratamiento & Control & Tratamiento 1 & Tratamiento 2 & Control & Tratamiento 1 & Tratamiento 2 \\
\hline Berula erecta & & $X$ & & & $x$ & \\
\hline Bidens aurea & $X$ & & & & & \\
\hline Cyperus esculentus & $x$ & $X$ & $X$ & & & \\
\hline Cyperus niger & $x$ & $x$ & $x$ & $X$ & $x$ & $X$ \\
\hline Eleocharis montevidensis & $x$ & $x$ & $X$ & $X$ & $x$ & $X$ \\
\hline Epilobium ciliatum & & $x$ & & $x$ & & \\
\hline Eupatorium rugosum & $X$ & $x$ & $X$ & $X$ & $X$ & $X$ \\
\hline Galium trifidum & & & $\mathrm{x}$ & & $x$ & \\
\hline Gnaphalium americanum & $X$ & $x$ & $x$ & $X$ & & \\
\hline Hydrocotyle verticillata & $x$ & $x$ & $X$ & $X$ & $x$ & $\mathrm{X}$ \\
\hline Lythrum salicaria & $x$ & & & $x$ & & \\
\hline Mimulus glabratus & & $X$ & $X$ & & $X$ & $X$ \\
\hline Poaceae 1 (Gramineae) & $X$ & $x$ & & $X$ & $x$ & \\
\hline Poaceae 2 (Gramineae) & $x$ & & & $x$ & & \\
\hline Polygonum hydropiperoides & $x$ & $X$ & $X$ & $X$ & $x$ & $X$ \\
\hline Sagittaria latifolia & $x$ & $x$ & $x$ & $X$ & $x$ & $X$ \\
\hline Schoenoplectus americanus & $x$ & $x$ & $x$ & $X$ & $x$ & $X$ \\
\hline Solanum americanum & & $x$ & & & $X$ & \\
\hline
\end{tabular}

Durante el primer año del experimento se evaluó mensualmente la cobertura de las especies y la radiación fotosintética, la cual se midió con un Apogee Instruments Quantum Meter Basic Model BQM-SUN. Durante todo el experimento se evaluó el número de tallos y alturas de Phragmites australis y la altura de Schoenoplectus americanus.

Los datos de altura (cm), biomasa aérea (g) de Schoenoplectus americanus y Phragmites australis, el número de retoños y rizomas (g) de $P$. australis y la radiación fotosintéticamente activa $(\mu \mathrm{mol} \mathrm{m}-2 \mathrm{~s}-1)$ incidente en las unidades experimentales se analizaron mediante análisis de varianza seguido de comparaciones múltiples de medias con la prueba de Tukey, utilizándose el paquete estadístico JMP 3.2.2.

\section{Resultados}

De abril de 2009 a octubre de 2010 se logró el establecimiento de 17 especies además de la dominante Schoenoplectus americanus. En el mes de abril sólo se encontraron ocho especies, en mayo diez, en junio 16, en julio 15 (presentó mortalidad de algunos individuos de especies raras), en agosto 14, en octubre seis, en noviembre tres y en diciembre y enero seis. Las partes aéreas de muchas especies se secan en invierno y otras son especies anuales, por ello su número varió a lo largo del año. A partir del mes de marzo se incrementó el número de especies, repitiéndose el patrón en los meses siguientes (Cuadro 1).

Hidrocotyle verticilata se registró durante todo el experimento y en todas las unidades experimentales, mientras que Sagittaria latifolia también se registró en todas las unidades experimentales pero sólo en algunos meses del año, ya que su parte aérea muere en invierno. Algunas especies como Cyperus niger disminuyeron su abundancia en el transcurso de los meses y otras como C. esculentus sólo se presentaron en unos pocos meses. Especies que aumentaron su cobertura fueron Eleocharis montevidensis, Epilobium ciliatum y Eupatorium rugosum que se encontraron con coberturas del $20 \%$ en las unidades experimentales control en la primera temporada y la incrementaron hasta un $30 \%$ en la segunda. Polygonum hydropiperoides fue una de las pocas especies que se presentaron en un mayor número de unidades experimentales conforme avanzó el experimento pasando de dos unidades experimentales en 2009 a cinco en el 2010 (Cuadro 2).

Se registraron diferencias entre el tratamiento control y los tratamientos de fertilización $\left(F_{(1,33)}=6.046, P=0.0038\right)$ para la radiación fotosintéticamente activa, desde el mes junio de 2009. Las evaluaciones de este parámetro durante 2010 no mostraron diferencias entre los tratamientos uno y dos de fertilización $\left(F_{(1,33)}=0.0001, P=0.9906\right)$, sólo con el tratamiento control $\left(F_{(1,33)}=9.036, P=0.0031\right)$, al mismo tiempo que el efecto de presencia o ausencia de Phragmites australis (en las parcelas divididas) no tuvo efecto $\left(F_{(1,33)}=\right.$ $0.193, P=0.6616$ ).

Schoenoplectus americanus. Para S. americanus, de los análisis de varianza se desprende que hubo diferencias para la altura máxima, la altura promedio de tallos y el número de tallos $\left(F_{(1,33)}=74.687, P<0.0001\right)$ y para el porcentaje de cobertura $\left(F_{(1,33)}=8.522, P=0.0118\right)$. La prueba de Tukey 


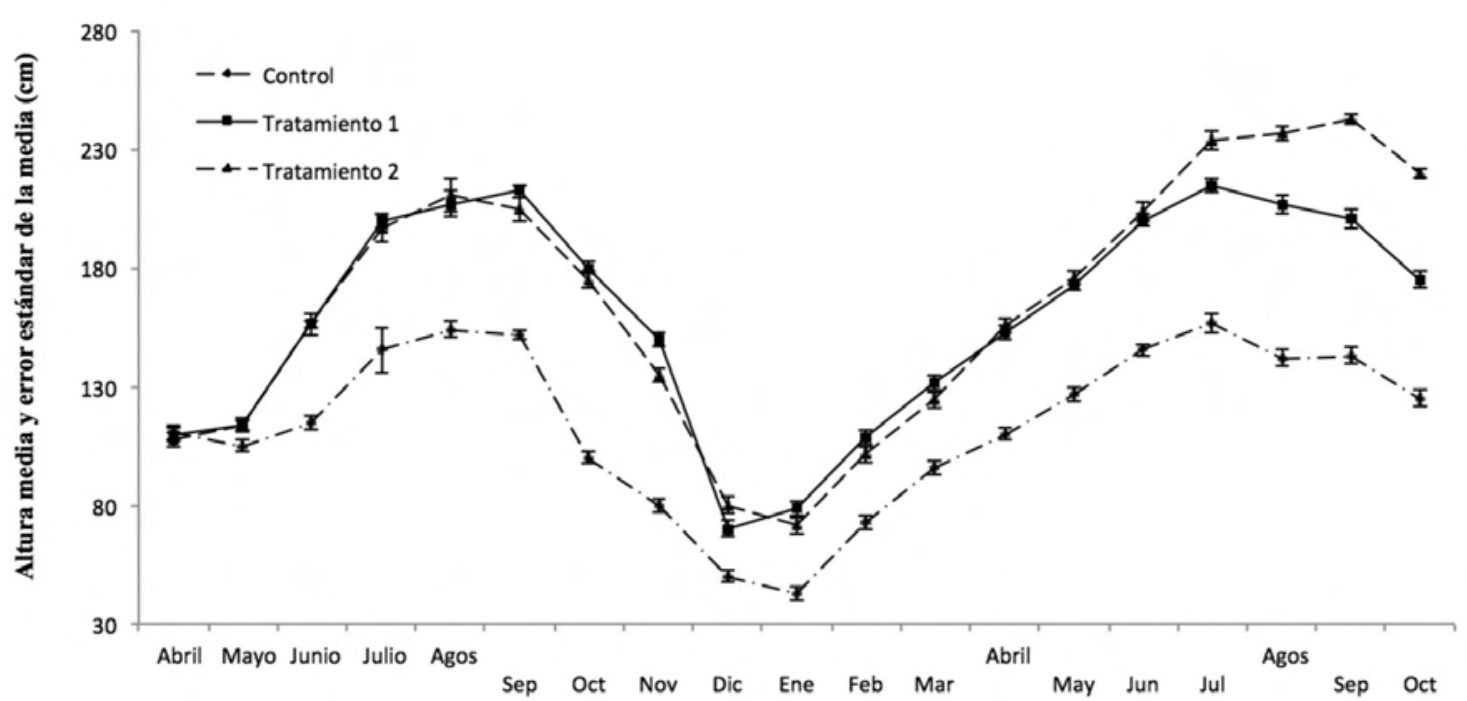

Figura 1. Altura media y error estándar de la media de Schoenoplectus americanus de abril de 2009 a octubre de 2010.

indicó que para la altura máxima de tallos fue el tratamiento dos (fertilización en dos temporadas) el que produjo un rendimiento estadísticamente mejor que los otros dos tratamientos (Cuadro 3). Un patrón similar para la altura promedio de los tallos y el número de tallos se obtuvo para las evaluaciones del año 2010. En el año anterior (2009) también el tratamiento uno (fertilización primera temporada) produjo un rendimiento estadísticamente mejor que el tratamiento control.

La adición de nutrimentos al finalizar el experimento favoreció a Schoenoplectus americanus, lo cual se reflejó en la altura de sus tallos (Figura 1, Cuadro 3 ) y en el número de tallos en las dos temporadas evaluadas. Sin embargo, al inicio del experimento la adición de nutrimentos favoreció al incremento del porcentaje de biomasa de las gramíneas, por lo que las unidades experimentales que no se les adicionó nutrimentos presentaron mayor cobertura de S. americanus. Esta situación cambió en la segunda temporada de crecimiento, ya que en los meses de diciembre y enero el rebrote de $S$. americanus fue rápido en los tratamientos con adición de nutrimentos y logró dominar el dosel. En el tratamiento control $S$. americanus mostró menor crecimiento lo que permitió que las otras especies nativas rebrotaran.

Cuadro 3. Respuesta de Schoenoplectus americanus a los distintos tratamientos de fertilización. Los datos son medias y desviaciones estándar. * Diferencias significativas de las medias de la prueba de Tukey

\begin{tabular}{|c|c|c|}
\hline \multirow[b]{2}{*}{ Tratamiento } & \multicolumn{2}{|c|}{ Schoenoplectus americanus } \\
\hline & $\begin{array}{l}\text { Altura del tallo } \\
\text { más alto }(\mathrm{cm})\end{array}$ & $\begin{array}{l}\text { Biomasa } \\
\text { aérea (g) }\end{array}$ \\
\hline 1 & $214.58 \pm 13.43$ & $637.86 \pm 177.99$ \\
\hline 2 & $233.40^{*} \pm 18.11$ & $869.17^{*} \pm 349.69$ \\
\hline C & $167.27 \pm 9.04$ & $290.00 \pm 62.67$ \\
\hline
\end{tabular}

A partir de agosto de la primera temporada de crecimiento, las partes aéreas de la mayoría de las especies empezaron a secarse por lo que la altura y número de tallos de Schoenoplectus americanus descendió. La altura máxima en los tratamientos control se registró en julio de 2009 alcanzando $187 \mathrm{~cm}$ y en agosto de $2010180 \mathrm{~cm}$. Mientras que la altura máxima en los tratamientos con adición de nutrimentos fue de $270 \mathrm{~cm}$ en agosto de 2009 y en el 2010 el tratamiento uno fue de $240 \mathrm{~cm}$ en julio y el tratamiento dos de $260 \mathrm{~cm}$ en septiembre. En cuanto a las alturas promedio de los tallos de $S$. americanus los valores máximos se registraron en agosto del $2009 \mathrm{con} 154 \mathrm{~cm}$ y en julio de 2010 con $156 \mathrm{~cm}$ (Figura 1).

El número de tallos de Schoenoplectus americanus, en las unidades experimentales del tratamiento control para la primera temporada de crecimiento fue de 178 tallos en promedio, disminuyendo para la segunda temporada a 160 tallos. Durante la primera temporada de crecimiento, en los tratamientos 1 y 2 , el promedio de tallos fue de 220 por unidad experimental, y en la segunda para el tratamiento 1 fue de 248 tallos y de 300 para el tratamiento 2, incrementándose la formación de tallos cerca del $30 \%$ con la adición de nutrimentos. Schoenoplectus americanus respondió a la adición de nutrimentos incrementando su biomasa aérea $\left(F_{(1,33)}=69.477, P=<0.0001\right)$, la prueba de Tukey indica que en el tratamiento 2 se produjo más biomasa aérea que en los otros tratamientos. Mientras que el efecto de la presencia de Phragmites australis no fue significativo en las variables evaluadas: altura máxima, altura promedio, porcentaje de cobertura $\left(F_{(1,33)}=0.0163, P=0.7378\right)$ y biomasa aérea $\left(F_{(1,33)}=0.001, P=0.9677\right.$.

Phragmites australis. Para P. australis se encontraron diferencias significativas entre tratamientos para la altura $\left(F_{(1,33)}\right.$ $=13.209, P=0.0001)$, con los datos finales del experimento 


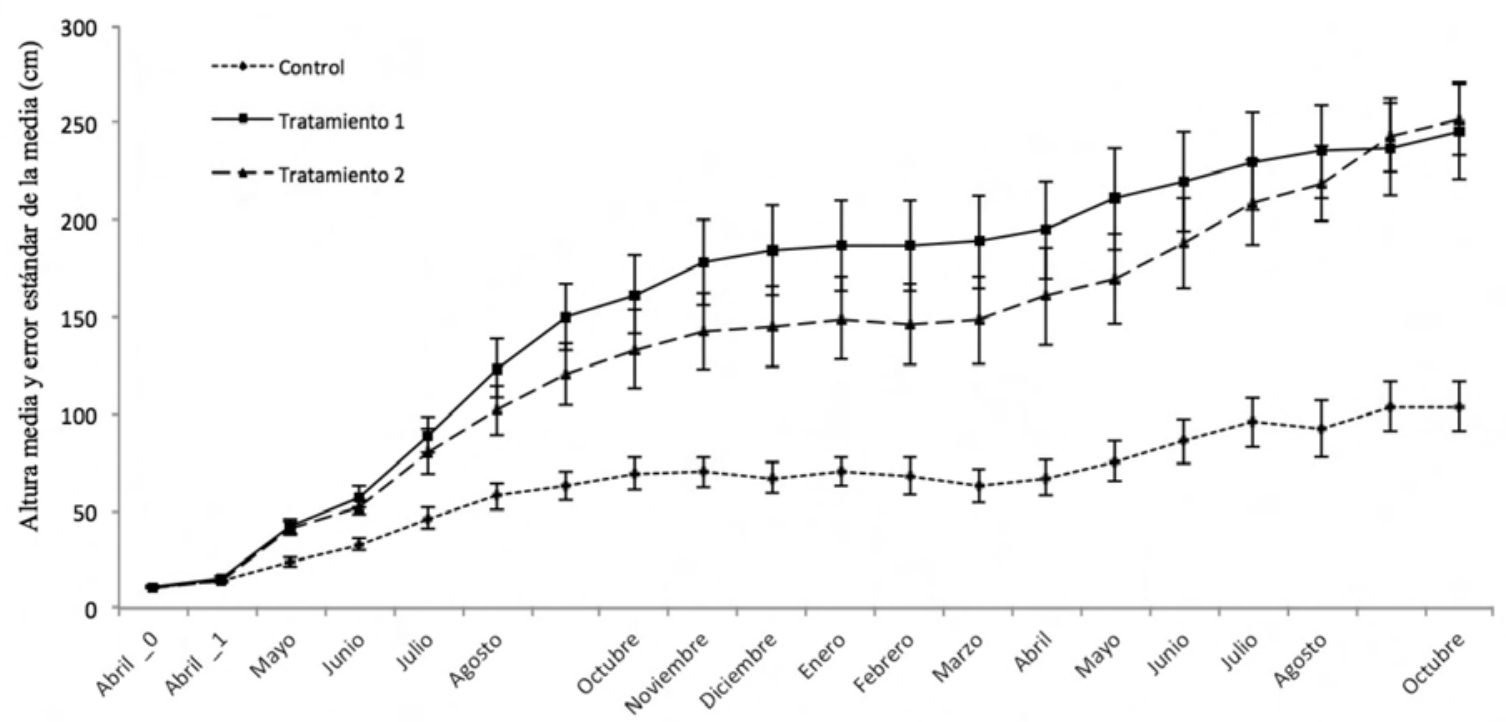

Figura 2. Alturas promedio y error estándar de la media de Phragmites australis por tratamiento de abril de 2009 a octubre de 2010

(abril 2011). La prueba de Tukey indica que los tratamientos 1 y 2 produjeron un rendimiento estadísticamente mejor que el tratamiento control, por lo que la adición de nutrimentos favorece a $P$. australis en términos del crecimiento de tallos.

A partir de mayo de la primera temporada de crecimiento (2009) la adición de nutrimentos favoreció el crecimiento del tallo de Phragmites australis. Sin embargo en 2010, cuando inicia el segundo periodo de fertilización, ya no se encontraron diferencias significativas $\left(F_{(1,33)}=0.007, P=\right.$ 0.6571 ) entre los tratamientos 1 y 2 , lo cual se mantiene hasta el final del experimento.

El crecimiento de Phragmites australis fue lento al inicio del experimento (Figura 2), siendo hasta el mes de marzo de 2010, es decir el inicio de la segunda temporada, cuando aumentó la talla de los individuos de esta especie. Los individuos en las unidades experimentales del tratamiento 1, redujeron ligeramente su crecimiento en los meses de agosto y septiembre, para aumentarlo en el mes de octubre, mientras que el tratamiento 2 desde agosto aceleró su crecimiento.

En cuanto a los retoños de Phragmites australis, su número se incrementó de abril a agosto y en los meses de septiembre y octubre fue cuando se registró la mayor mortan- dad, para aumentar de nuevo en noviembre, siendo durante el mes de enero de 2010 cuando se encontró la mayor cantidad de retoños promedio (8). Entre febrero y mayo murieron algunos retoños, para luego volver a incrementar su número, repitiéndose el patrón para el siguiente periodo de crecimiento y hasta finaliza el experimento. Al finalizar el experimento en los tratamientos control el número de retoños osciló de 2 a 6 , mientras que el tratamiento 1 fue de 2 a 9 y finalmente el tratamiento 2 fue donde se registraron hasta 13 retoños (Figura 3, Cuadro 4), encontrándose diferencias significativas entre el control y los dos tratamientos de fertilización $\left(F_{(1,33)}=9.489, P=0.0007\right)$.

Al finalizar el experimento, los análisis de varianza mostraron diferencias significativas para el peso final de rizomas $\left(F_{(1,3)}=13.326, P=<0.0001\right)$ y para la biomasa aérea $\left(F_{(1,33)}=9.644, P=0.006\right)$ de Phragmites australis. La prueba de Tukey mostró que para los rizomas y la biomasa aérea el tratamiento 2 tuvo un mayor incremento con respecto a los otros dos tratamientos.

La cosecha de biomasa aérea de Schoenoplectus americanus como un mecanismo de disturbio no afectó a Phragmites australis. Sin embargo $S$. americanus, en respuesta a la cosecha de sus hojas, aceleró su crecimiento $\left(F_{(1,33)}\right.$

Cuadro 4. Respuesta de Phragmites australis a los distintos tratamientos de fertilización. Los datos son medias y desviaciones estándar. * Diferencias significativas de las medias de la prueba de Tukey.

\begin{tabular}{ccccc}
\hline Tratamiento & $\begin{array}{c}\text { Altura del tallo } \\
\text { más alto }(\mathrm{cm})\end{array}$ & $\begin{array}{c}\text { Phragmites australis } \\
\text { Túmero de } \\
\text { Tallos }\end{array}$ & $\begin{array}{c}\text { Biomasa } \\
\text { aérea }(\mathrm{g})\end{array}$ & $\begin{array}{c}\text { Biomasa de } \\
\text { rizomas }(\mathrm{g})\end{array}$ \\
\hline 1 & $239.73^{*} \pm 87.43$ & $6.27^{*} \pm 2.28$ & $98.61^{*} \pm 88.89$ & $31.09^{*} \pm 17.85$ \\
$\mathrm{C}$ & $247.50^{*} \pm 60.43$ & $6.80^{*} \pm 2.57$ & $143.61^{*} \pm 84.16$ & $59.72^{*} \pm 31.41$ \\
& $118.91 \pm 37.28$ & $3.18 \pm 1.17$ & $11.40 \pm 11.25$ & $12.78 \pm 7.89$ \\
\hline
\end{tabular}




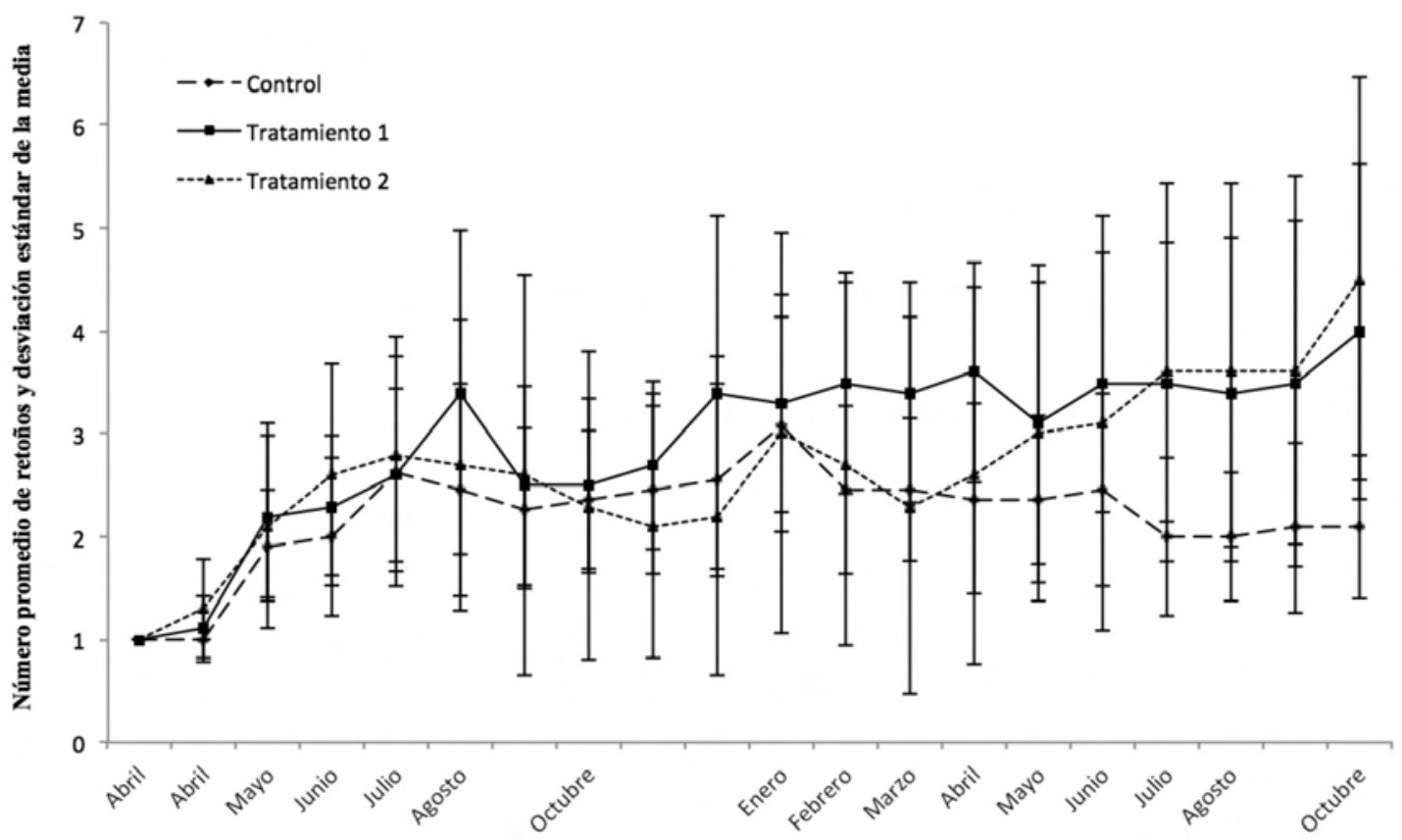

Figura 3. Número promedio de retoños y desviación estándar de la media de Phragmites australis por tratamiento de abril a de 2009 a octubre de 2010

$=5.215, \quad P=0.0020)$ en los tratamientos de adición de nutrimentos, pero no en el control, en donde la remoción de su biomasa favoreció principalmente a las especies de Cyperus.

\section{Discusión}

El hecho de que Phragmites australis no fuera capaz de dominar estos humedales experimentales en el corto plazo, sugiere que el incremento de nutrimentos por sí sólo no es suficiente para permitir el proceso de invasión como se ha reportado (Miao y Bazzaz, 1990; Verhoeven et al., 1993; Verhoeven et al., 1996) y sugiere que otros mecanismos deben de operar en conjunto. En nuestros experimentos, Schoenoplectus americanus respondió vigorosamente a la adición de nutrimentos y por lo tanto no fue desplazada por $P$. australis. La respuesta de $S$. americanus a la adición de nutrimentos es congruente con resultados obtenidos anteriormente (Escutia-Lara et al., 2010). En el campo $P$. australis alcanza alturas considerables y forma manchones prácticamente monoespecíficos (Bruce et al., 1995; Sanleón et al., 1999; Rogers y Hartemink, 2000). Llama la atención que en este experimento no se logró formar una cobertura lo suficientemente densa como para sombrear a las otras especies, al contrario, fue $S$. americanus la que desarrolló una cobertura más densa en los tratamientos fertilizados. Lo anterior sugiere que para que $P$. australis llegue a desplazar a las especies nativas se requiere de algún patrón de disturbio, antrópico o natural, que reduzca la competencia con especies nativas dominantes como S. americanus o que se requiere de muchos propágulos y tiempo suficiente para que haya unos pocos eventos de invasión exitosos. Un posible factor de disturbio sería el fuego, que se sabe altera la dinámica de las especies nativas en nuestro sistema modelo, los humedales de la Mintzita (Escutia-Lara et al., 2009).

Lo anterior se refuerza si se considera que al momento que Phragmites australis comenzó su desarrollo en las unidades experimentales, Schoenoplectus americanus ya llevaba cerca del año de crecimiento y había creado un dosel cerrado. Esto coincide con diversos estudios que han mostrado que el proceso de invasión en humedales se ve favorecido por factores que reducen o eliminan la competencia con las especies nativas, como sería el caso de la remoción de la vegetación (Gaudet y Keddy, 1995; Nernberg y Dale, 1997).

En el manantial de la Mintzita en Michoacán, Phragmites australis ha incrementado su cobertura en los últimos años (Escutia-Lara obs.), lo que coincide con un aumento en el número y frecuencia de incendios provocados por los pobladores locales. Es de esperarse que después de un incendio P. australis y Schoenoplectus americanus compitan por el espacio y los nutrimentos disponibles. Sin embargo, a diferencia de este experimento, inician el crecimiento al mismo tiempo, pudiendo ser éste un factor que beneficie a $P$. australis, ya que esta especie desarrolla su parte aérea en cualquier época del año, mientras que $S$. americanus al finalizar el año su parte aérea muere como se cuantificó en el presente estudio.

Es poca la información que se tiene sobre cómo Phragmites australis desplaza a otras especies hasta volver el sitio monoespecífico. Sin embargo, estos resultados permiten 
sugerir que uno de los factores importantes aparte del incremento en los nutrimentos son los disturbios, puesto que de no presentarse alguno, P. australis si se desarrolla mejor con la adición de nutrimentos pero no se convierte en una especie invasora aunque es una especie no nativa, por lo que al presentarse algún disturbio existen las condiciones adecuadas para que se desarrolle y desplace a las otras especies nativas (Bakker y Wilson, 2001).

$\mathrm{La}$ adición de nutrimentos para el incremento en la altura de los tallos para Schoenoplectus americanus es necesaria anualmente, como se observó en los dos tratamientos de adición de nutrimentos. Mientras que para Phragmites australis esto no es un requisito, su crecimiento no depende de un segundo aporte de nutrimentos para la altura y el número de tallos, pero sí para el incremento en la biomasa aérea y de rizomas.

Phragmites australis por ser una planta perennifolia, durante diciembre y enero se beneficia desarrollando más tallos e incrementando el número de retoños, mientras que las demás especies anuales como Schoenoplectus americanus rebrotan. Sin embargo, en condiciones naturales esta última será capaz de competir por los nutrimentos sin ser desplazada por $P$. australis siempre y cuando no se presente algún disturbio, como lo demuestra este estudio donde se mantuvo la composición y estructura original antes de introducir a $P$. australis. Los resultados sugieren que la competencia intraespecífica es más fuerte que la interespecífica en ausencia de disturbio.

\section{Agradecimientos}

Este proyecto fue financiado por la Universidad Nacional Autónoma de México por medio del proyecto PAPIIT IN203608 y CONACYT (SEP-CONACYT-2008-0101335), así mismo agradecemos las facilidades brindadas para la instalación del experimento en el CIEco-UNAM campus Morelia, a la UMSNH, Facultad de Biología y a todos los que colaboraron haciendo posible el desarrollo de dicho proyecto.

\section{Literatura citada}

Aerts R. y Chapin F.S. 1999. The mineral nutrition of wild plants revisited: a re-evaluation of processes and patterns. Advances in Ecological Research 30:1-67.

Aerts R. y de Caluwe H. 1994. Nitrogen use efficiency of Carex species in relation to nitrogen supply. Ecology 75:2362-2372.

Alpert P., Bone E. y Holzapfel C. 2000. Invasiveness, invasibility and the role of environmental stress in the spread of non-native plants. Perspectives in Plant Ecology, Evolution and Systematics 3:52-66.

Berendse F. y Aerts R. 1987. Nitrogen use efficiency: a biologically meaningful definition? Functional Ecology 1:293-296.

Bakker J. y Wilson S. 2001. Competitive abilities of introduced and native grasses. Plant Ecology 157:119-127.
Boar R.R. 1996. Temporal variations in the nitrogen content of Phragites australis (Cav.) Trin. ex Steud. from a shallow fertile lake. Aquatic Botany 55:171-181

Boyd C.E. 1970. Production, mineral accumulation and pigment concentrations in Typha latifolia and Scirpus americanus. Ecology 51:285-290.

Bruce K.A., Cameron G.N. y Harcombe P.A. 1995. Initiation of a new woodland type on the Texas coastal prairie by the Chinese tallow tree (Sapium sebiferum (L.) Roxb.) Bulletin of the Torrey Botanical Club 122:215-225.

D'Antonio C.M. 1993. Mechanisms controlling invasion of coastal plant communities by the alien succulent Carpobrotus edulis. Ecology 74:83-95.

D’Antonio C.M. y Vitousek P.M. 1992. Biological invasions by exotic grasses, the grass/fire cycle, and global change. Annual Review of Ecology, Evolution, and Systematics 23:63-87.

Escutia-Lara Y. 2008. Composición y dominancia de especies hidrófitas emergentes y captura de nitrógeno y fósforo en humedales de agua dulce en Michoacán, México. Tesis de Maestría, Facultad de Biología, Universidad Michoacana de San Nicolás de Hidalgo, Morelia. 70 pp.

Escutia-Lara Y., Gómez-Romero M. y Lindig-Cisneros R. 2009. Nitrogen and phosphorus effect on Typha domingensis Presl. rhizome growth in a matrix of Schoenoplectus americanus (Pers.) Volkart ex Schinz and Keller. Aquatic Botany 90:74-77.

Escutia-Lara Y., de la Barrera E., Martínez-de la Cruz Y. y Lindig-Cisneros R. 2010. Respuesta a la adición de nitrógeno y fósforo en el crecimiento de Typha domingensis y Schoenoplectus americanus. Boletín de la Sociedad Botánica de México 87:83-87.

Gaudet C.L. y Keddy P.A. 1995. Competitive performance and species distribution in shoreline plant communities: a comparative approach. Ecology 76:280-291.

Goldberg D.E. 1987. Neighborhood competition in an old-field plant community. Ecology 68:1211-1223.

Goldberg D.E. 1990. Components of resource competition in plant communities. En: Grace J.B. y Tilman D. Eds. Perspectives on Plant Competition, pp. 27-49, Academic Press, San Diego.

Gordon D.R. 1998. Effects of invasive, non-indigenous plant species on ecosystem processes: lessons from Florida. Ecological Applications 8:975-989.

Gosselink J.G. y Turner R.E. 1978. The role of hydrology in freshwater wetland ecosystems. En: Good R.E, Whigham D.F. y Simpson R.L. Eds. Freshwater Wetlands: Ecological Processes and Management Potential, pp. 63-78, Academic Press, Nueva York.

Howard T.G. y Goldberg D.E. 2001. Competitive response hierarchies for germination, growth, and survival and their influence on abundance. Ecology 82:979-990.

Huenneke L.F. y Thomson J.K. 1995. Potential interference between a threatened endemic thistle and an invasive nonnative plant. Conservation Biology 9:416-425

Hwang Y.H., Fan C.W. y Yin M.H. 1996. Primary production and chemical composition of emergent acuatic macrophytes, Schoenoplectus mucronatus ssp. Robustus and Sparaginum fallax, in Lake Yuan-Tang, Taiwan. Botanical Bulletin of Academia Sinica 37:265-273.

Hwang Y.H., Liou C.F. y Weng I.S. 2000. Nutrient dynamics of two aquatic angiosperms in an alpine lake, Taiwan. Botanical Bulletin of Academia Sinica 41:275-282. 
Ksenofontova T. 1988. Morphology, production and mineral contents in Phragmites australis in different water bodies of the Estonian SSR. Folia Geobotanica et Phytotaxonomica 23:17-43.

León B., Cano A. y Young K.R. 1995. La flora vascular de los pantanos de Villa, Lima, Perú: Adiciones y guía para las especies comunes. Publicaciones del Museo de Historia Natural - UNMSM. (B) 38:1-39.

Lindig-Cisneros R. y Zedler J.B. 2002a. Relationships between canopy complexity and germination microsites for Phalaris arundinacea L. Oecologia 133:159-167.

Lindig-Cisneros R. y Zedler J.B. 2002b. Phalaris arundinacea seedling establishment: effects of canopy complexity in fen, mesocosm, and restoration experiments. Canadian Journal of Botany 80:617-624.

Mack R.N., Simberloff D., Lonsdale W.M., Evans H., Clout M. y Bazzaz F. 2000. Biotic invasion: causes, epidemiology, global consequences, and control. Ecological Applications 10:689710.

Martínez-de la Cruz Y. 2010. Efecto de la adición de Nitrógeno y Fósforo en el crecimiento y respuestas fisiológicas de Schoenoplectus americanus, Typha domingensis y Phragmites australis. Tesis de Licenciatura, Facultad de Biología, Universidad Michoacana de San Nicolás de Hidalgo, Morelia. 55 pp.

Meekins J.F. y McCarthy B.C. 1999. Competitive ability of Alliaria petiolata (Garlic Mustard, Brassicaceae), an invasive, nonindigenous Forest Herb. International Journal of Plant Sciences 160:743-752.

Miao S.L. y Bazzaz, F.A. 1990. Responses to nutrient pulses of two colonizers requiring different disturbance frequencies. Ecology 71: 2166-2178.

Mitsch W.J. y Gosselink J.G. 1993. Wetlands. John Wiley and Sons, Nueva York.

Montoya T. 1984. Algas de la Laguna de Villa (Lima): Cyanophyta, Chlorophyta y Chrysophyta (Xanthophyceae). Boletín de Lima 32:49-62.

Moore D.R.L. y Keddy, P.A. 1988. Effects of a water-depth gradient on the germination of lakeshore plants. Canadian Journal of Botany 66:548-552.

Nernberg D. y Dale M.R.T. 1997. Competition of five native prairie grasses with Bromus inermis under three moisture regimes. Canadian Journal of Botany 75:2140-2145.

Nuñez-Escobar R. 2007. El suelo como medio natural en la nutrición de cultivos. En: González-Alcántar G. y Trejo-Téllez L.I. Coords. Nutrición de Cultivos. pp. 93-157 Colegio de Posgraduados/Mundiprensa, México, D.F.

Peltzer D.A. y Köchy M. 2001. Competitive effects of grasses and woody plants in mixed-grass prairie. Journal of Ecology 89:519-527.

Recibido: 9 de agosto, 2011

Aceptado: 23 de febrero, 20112
Reader R.J. y Bonser S.P. 1993. Control of plant frequency on an environmental gradient: effects of abiotic variables, neighbours, and predators on Poa pratensis and Poa compressa (Gramineae). Canadian Journal of Botany 71:592-597.

Rogers H.M. y Hartemink A.E. 2000. Soil seed bank and growth rates of an invasive species, Piper aduncum, in the lowlands of Papua New Guinea. Journal of Tropical Ecology 16:243-251.

SanLeón D.G., Izco J. y Sánchez J.M. 1999. Spartina patens as a weed in Galician saltmarshes (NW Iberian Peninsula). Hydrobiologia 415:213-222.

Thompson K., Hodgson J.G., Grime J.P. y Burke M.J.W. 2001. Plant traits and temporal scale: evidence from a 5-year invasion experiment using native species. Journal of Ecology 89:10541060 .

van der Valk, A.G. y Davis C.B. 1978. The role of the seed bank in the vegetation dynamics of prairie glacial marshes. Ecology 59:322-335.

Verhoeven J.T.A., Kemmers R.H. y Koerselman W. 1993. Nutrient enrichment of freshwater wetlands. En: Vos C.C. y Opdam P. Eds. Landscape Ecology of a Stressed Environment, pp. 33-59, Chapman \& Hall, Londres.

Verhoeven J.T.A., Koerselman W. y Meuleman A.F.M. 1996. Nitrogen- or phosphorus-limited growth in herbaceous, wet vegetation: relations with atmospheric inputs and management regimes. Trends in Ecology and Evolution 11:494-497.

Vitousek P.M., Walker L.R., Whittacker L.D., Mueller-Dombois D. y Matson P.A. 1987. Biological invasion by Myrica faya alters ecosystem development in Hawaii. Science 238:802-804.

Ulrich K.E. y Burton T.M. 1985. The effects of nitrate, phosphate and potassium fertilisation on growth and nutrient uptake patterns of Phragmites australis (Cav.) Trin. ex Steudel. Aquatic Botany 21:53-62.

Lloyd-Reilley J. y Maher S.D. 2011. E. "Kika" de la Garza Plant Materials Center, Year 2010 Progress Report of Activities Plant Materials Annual Report of Activities United States Deparment of Agriculture.Kingsville. Disponible en linea: <www.plantmaterials.nrcs.usda.gov/pubs/stpmcra10305.pdf >

Weigelt A., Steinlein T. y Beyschlag W. 2002. Does plant competition intensity rather depend on biomass or on species identity? Basic and Applied Ecology 3:85-94.

Weihe P.E. y Neely R.K. 1997. The effects of shading on competition between purple loosestrife and broad-leaved cattail. Aquatic Botany 59:127-138.

Young K.R. 1998. El ecosistema. En: Cano A.y Young K. Eds. Los Pantanos de Villa: Biología y Conservación, pp. 3.20 Museo de Historio Natural, Universidad Nacional Mayor de San Marcos, Lima. 